Revue d'histoire de l'Amérique française

REVUE D.HISTOIRE DE L'AMÉRIQUE FRANÇAISE

\title{
Jean Nicollet dans le lac Supérieur et non dans le lac Michigan
}

\section{Marcel Trudel}

Volume 34, numéro 2, septembre 1980

URI : https://id.erudit.org/iderudit/303855ar

DOI : https://doi.org/10.7202/303855ar

Aller au sommaire du numéro

Éditeur(s)

Institut d'histoire de l'Amérique française

ISSN

0035-2357 (imprimé)

1492-1383 (numérique)

Découvrir la revue

Citer cet article

Trudel, M. (1980). Jean Nicollet dans le lac Supérieur et non dans le lac Michigan. Revue d'histoire de l'Amérique française, 34(2), 183-196.

https://doi.org/10.7202/303855ar d'utilisation que vous pouvez consulter en ligne.

https://apropos.erudit.org/fr/usagers/politique-dutilisation/ 


\title{
JEAN NICOLLET DANS LE LAC SUPÉRIEUR ET NON DANS LE LAC MICHIGAN
}

\author{
MARCEL TRUDEL \\ Département d'histoire \\ Université d'Ottawa
}

Selon la version traditionnelle, Jean Nicollet ${ }^{1}$ arrive au Canada en 1618 et va chez les Amérindiens apprendre puis pratiquer le métier d'interprète. Sur l'ordre de Champlain, à ce qu'on dit, il part des Trois-Rivières en 1634 pour la Huronie, d'où il entreprend une ambassade chez les Gens de Mer, dits aussi Ouinipigous ou Puants. Mais où sont ces Gens de Mer?

L'historien américain John Gilmary Shea est le premier à «découvrir» en 1852 que Nicollet s'est rendu en 1639 dans le lac Michigan et dans la baie Verte (aujourd'hui Green Bay): comme les Gens de Mer ou Puants ne parlaient, selon Shea, ni l'algonquin ni le huron et qu'il y aura dans le lac Michigan une baie des Puants fréquentée par des peuples de langue siouse, les Winnebagoes, ce sont eux, conclut Shea, que Nicollet est allé visiter et, ajoute l'historien, Champlain a fait erreur en situant son lac des Puants au nord des Grands Lacs'2. L'historien canadien-français Benjamin Sulte adopte cette même thèse vingt ans plus tard, en proposant toutefois l'année $1634-1635$ au lieu de $1639^{3}$. Il est suivi en 1881 par un autre his-

1 Nous écrivons Nicollet selon la manière adoptée par le Dictionnaire biographique $d u$ Canada et qui correspond aux signatures: l'interprète signait lui-même Nicollet; son frère Gilles, prêtre séculier qui fit du ministère au Canada de 1643 à 1647, signait aussi Nicollet; quant à son autre frère, le matelot Pierre, présent au pays en 1637 et de 1640 à 1647, on n'a de lui qu'une marque $P$.N. qu'il appose sur un document du 24 octobre 1640; ailleurs, il déclare habituellement ne savoir signer.

2 J.G. Shea, Discovery And Exploration of the Mississippi Valley (Redfield, New York, 1852), xx-xxi.

3 Â une époque où l'on parlait beaucoup de la découverte du Mississipi par Jolliet et Marquette, Sulte estime qu'on a négligé Nicollet; c'est pourquoi, il publie une étude dans le Journal de l'instruction publique, XVII (1873): 166s.; XVIII (1874): 28-32; il reprend cette étude dans ses Mélanges d'histoire et de littérature (éd. 1876), 411-451. Sulte revient à la charge en 1910 dans la Revue canadienne (VI: 148-155, 331-342, 409-420), «Jean Nicolet et la découverte du Wisconsin, 1634».

RHAF, vol. 34, no 2, septembre 1980 
torien américain, C.W. Butterfield, qui développe beaucoup plus longuement cette thèse d'un Nicollet découvreur du Michigan et du Wisconsin ${ }^{4}$. En 1905, Auguste Gosselin donne plus d'ampleur encore à cette thèse $\mathrm{S}^{5}$. Ce que, par la suite, les historiens ont repris, l'un répétant l'autre avec tout le respect pour l'autorité établié6.

Selon cette thèse traditionnelle, propre à flatter notre orgueil national, Nicollet entre donc dans le lac Michigan, se rend dans la baie des Puants, conclut la paix, pousse plus loin dans l'espoir de trouver la mer de Chine, descend la rivière aux Renards jusqu'à un village de Mascoutins; il n'était plus qu'à trois jours de voyage de la rivière Wisconsin qui se jette dans le Mississipi. Il revient enfin à Québec en 1635 faire rapport à Champlain, puis va s'installer aux Trois-Rivières.

Or, à la suite de diverses suggestions ${ }^{7}$ et parce que nous éprouvons le besoin de vérifier ce qui a été dit de la période des Cent-Associés, nous nous rendons compte qu'une large partie de cette version est à modifier: autorité responsable de l'ambassade, année de cette ambassade et, surtout, itinéraire qu'elle a suivi.

\section{L'unique source d'information}

Jean Nicollet avait laissé des mémoires, mais ils n'ont pas été retrouvés ${ }^{8}$, de sorte que les Relations des Jésuites demeurent la seule

4 C.W. Butterfield, History of the Discovery of the Northwest by John Nicolet in 1634 With a Sketch of His Life (Port Washington, N.Y., Kennikat Press, 1881; ix-113p., 20,5cm): voir, en particulier, les pages 35-74.

5 A. Gosselin, Jean Nicolet et le Canada de son temps, 1618-1642 (Québec, K.-Laflamme, 1905; viii-282p., 18cm.)

6 Nous avions nous-même jusqu'ici répété cette version traditionnelle dans nos oeuvres, Initiation à la Nouvelle-France, Introduction to New France, The Beginnings of New France, ainsi que dans l'introduction au volume premier du Dictionnaire biographique du Canada, ce même ouvrage qui, d'ailleurs, publie de Jean Hamelin un article conforme à cette même version traditionnelle (I: 527-529). Voilà donc notre coulpe battue...

7 Le 3 mai 1967, après la publication de l'article du Dictionnaire sur Jean Nicollet, l'historien américain Donald Chaput, de la Michigan Historical Commission, écrivit au professeur Hamelin pour lui signaler un article de Harry Dever, The Nicolet Myth, dans Michigan History, 50, 4 (December 1966): 318-322. Le point le plus important de cet article montrait que les historiens ne s'étaient pas souciés de suivre de près le texte des Relations, unique source d'information sur le voyage de Nicollet. Chaput profitait de sa lettre pour signaler l'absence de toute preuve d'un rôle qu'aurait joué Champlain dans la mission de Nicollet et pour établir que Nicollet est arrivé au Canada en 1619 et non en 1618. Or le volume du Dictionnaire était déjà publié. Jean Hamelin eut l'amabilité de me transmettre cette correspondance.

8 Relation de 1636, dans RJ, 9: 214 (en cet article, c'est l'édition Thwaites que nous utilisons). Toutefois, les mémoires auxquels fait allusion cette Relation ne portent que sur les Népissingues. 
et unique source historique qui puisse nous renseigner sur l'ambassade de Nicollet. Les Jésuites en parlent en trois endroits différents de leurs Relations: celle de 1640, dans laquelle le P. Paul Le Jeune énumère, dans l'ordre géographique de l'aller, les nations amérindiennes des Grands Lacs, en se fondant sur les connaissances que Nicollet a rapportées de son voyage ${ }^{9}$; à l'occasion du décès de Nicollet en 1642, le P. Barthélemy Vimont, auteur de la Relation de 1642-1643, raconte ce voyage chez les Gens de $\mathrm{Mer}^{10}$; dans celle de 1655-1656, le P. Jean de Quen donne des chiffres sur la grande assemblée tenue chez les Ouinipigous par l'ambassadeur ${ }^{11}$.

C'est donc avec assez peu d'éléments que nous pouvons tenter de connaître ce voyage. Dans quelle mesure les Relations nous les transmettent-elles avec fidélité? Nous n'avons rien pour en juger, sauf sur la date que l'on attribue à l'arrivée de Nicollet au Canada. La Relation de 1642-1643 donne 1618; or on vient de remarquer aux Archives publiques du Canada un acte de vente passé en France par Jean Nicollet, le 10 mai $1619^{12}$ : Jean Nicollet, fils de Thomas Nicollet et présent à cette transaction, vend une terre qu'il possède à Hainneville, près de Cherbourg. Il ne pouvait donc être au Canada en 1618-1619; peut-être est-il venu quand même en 1618, mais seulement pour une courte visite? En tout cas, son installation définitive au Canada ne peut être antérieure à 1619 .

\section{Un voyage qu'on ne peut dater}

Lorsqu'on veut fixer la date de son voyage chez les Gens de Mer ou Ouinipigous, on est bien plus embarrassé. Les Relations n'en donnent aucune date et ne nous fournissent aucun élément qui puisse fonder sérieusement une hypothèse. Elles nous apprennent que d'abord Nicollet passe deux ans chez les Algonquins de l'île aux Allumettes (sur la rivière dite alors rivière des Algonquins et, plus tard, rivière des Outaouais), d'où il les accompagne en une mission de paix chez les Iroquois; après quoi, il vit «huict ou neuf ans» chez les Népissingues, autour du lac de ce nom, entre la rivière des Outaouais et la baie Georgienne: ce qui nous reporte à 1629 ou

Relation de 1640, RJ, 18: 228-236. Le Jeune précise qu'il tient de Nicollet luimême «les noms de ces nations qu'il a visité lui-même pour la pluspart dans leur pays».

10 Relation de 1642-1643, RJ, 23: 276-278.

11 Relation de 1655-1656, RJ, 42: 222.

12 APC, Papiers Nicolet, MG 18, B 14. 
$1630^{13}$. Puis, la Relation ajoute que Nicollet, rappelé dans le SaintLaurent pour y servir de commis et d'interprète, "fut delegué» vers les Gens de Mer. Il aurait donc fait son voyage après 1629 ou 1630 , ce qui laisse un intervalle de douze ans jusqu'à sa noyade en novembre 1642, près de Québec.

Comme le voyage du Saint-Laurent aux Grands Lacs et le retour au Saint-Laurent nécessitent, dans les conditions de l'époque, une pleine année (départ au cours du printemps ou de l'été, puis retour au printemps ou l'été suivant), il faut tout de suite éliminer les années 1635-1642: de l'automne de 1635 au décès de novembre 1642, la présence de Nicollet dans la colonie du Saint-Laurent est constamment attestée par divers documents ${ }^{14}$.

Le voyage n'a pu avoir lieu non plus en 1634-1635, comme on le supposait jusqu'ici. Cette hypothèse se fondait sur le voyage que fait Nicollet en 1634 jusqu'à l'île aux Allumettes (sur la rivière des Outaouais), en compagnie du jésuite Jean de Brébeuf ${ }^{15}$. Parce qu'on n'avait ensuite retrouvé 'Nicoliet dans la colonie du SaintLaurent qu'en août 1635, on avait conclu que de l'île aux Allumettes il avait poursuivi sa route pour aller remplir sa mission d'ambassade. Mais alors, comment expliquer que Nicollet, dont la mission se fait à partir de la Huronie (selon l'affirmation même de la Relation), se sépare de Brébeuf qui lui aussi s'en va en Huronie? C'est que tout simplement, en 1634 , Nicollet ne poursuit pas le voyage

13 Voir là-dessus la notice nécrologique que rédige le jésuite Barthélemy Vimont, dans la Relation de 1642-1643, RJ, 23: 274-276. C'est en ce séjour chez les Népissingues que Nicollet aurait eu d'une Amérindienne de cette nation une fille, appelée Madeleine-Euphrosine: le 18 janvier 1642, elle est marraine d'un sauvage chez les Ursulines de Québec; le 21 novembre 1642, elle épouse à Québec Jean Leblanc dit Lecourt, dont elle aura au moins cinq enfants; le 22 février 1663, elle se remarie à Elie Dusceau dit Lafleur dont elle aura quatre autres enfants; le recensement de 1666 lui donne 35 ans.

14 Nicollet est aux Trois-Rivières en décembre 1635, en janvier et en mai 1636, selon les actes de parrainage dans les registres des baptêmes et selon les Relations (1636, RJ, 8:246, 257, 264; 9: 125); il y est au printemps de 1637 (Relation de 1637, RJ, 12: 162); il se fait concéder une terre le 23 mai, il est témoin à une déclaration du 22 août, à une prise de possession de terre le 6 octobre, à un mariage le lendemain, à un contrat de mariage le 22 du même mois, il est parrain les 18 novembre et 18 décembre. En 1638, il est parrain les 24 janvier et 19 mars, il assiste à la prise de possession d'une terre le 16 août. En 1639, il est parrain les 9 janvier, 4 mars, 16, 18, 20 et 30 juillet, ainsi que le 7 décembre: ce qui élimine l'ancienne hypothèse de Shea sur une ambassade accomplie en 1639 et que, d'ailleurs, Sulte n'avait pas eu de difficulté à rejeter. Nicollet est toujours présent aux Trois-Rivières en 1640: il est parrain le 6 janvier, il assiste à un contrat de mariage le 2 septembre et il est de nouveau parrain le 24 décembre (voir aussi la Relation de 1640-1641, RJ, 20: 290). En juin et en août 1641, il est aussi aux Trois-Rivières: voir le contrat de mariage du 23 août et la Relation citée, RJ, 21 : 38 . Enfin, en 1642, il est parrain le 29 septembre et il se noie en novembre suivant.

15 Relation de 1634-1635, RJ, 8: 100. On écrit: «Jean Nicolet, en son voyage qu’il fit avec nous jusques à l'Isle souffrit aussi tous les travaux d'un des plus robustes Sauvages». 
au-delà de l'île aux Allumettes, il redescend aux Trois-Rivières: le 3 août 1634, Champlain qui est rentré à Québec après un voyage aux Trois-Rivières, raconte qu'il y a rencontré «un truchement François pour la nation Algonquine venant d'avec les Hurons» et que, selon cet interprète, le jésuite Brébeuf a bien souffert pendant son voya$\mathrm{ge}^{16}$. Cet interprète des Algonquins qui, à l'île aux Allumettes, se sépare du groupe des Hurons pour revenir au Saint-Laurent, ne peut être que Jean Nicollet, dont on sait qu'il s'est séparé du jésuite Brébeuf à cette étape. La mission de Nicollet en 1634 était seulement d'accompagner les missionnaires jusqu'à l'endroit le plus difficile à franchir, la célèbre barrière établie par les Algonquins de l'île aux Allumettes pour bloquer le passage aux Français, qu'ils fussent missionnaires ou laïques: Nicollet qui avait servi deux ans chez ces Algonquins, était l'homme qu'il fallait pour faciliter ce passage.

\section{De qui Nicollet a-t-il reçu sa mission?}

Éliminé $1634-1635$, il nous reste l'intervalle $1629-1634$ pour situer le voyage aux Gens de Mer. Nicollet l'aurait-il fait en 16331634? Les biographes affirment d'ordinaire que c'est Champlain, revenu en 1633 à Québec, qui aurait délégué Nicollet, et, pour sa part, Auguste Gosselin met beaucoup d'émotion dans le récit qui nous montre Nicollet remettant son rapport à Champlain ${ }^{17}$. Malheureusement, aucune pièce d'archives n'atteste l'authenticité ni de cette scène ni de cette délégation. Nulle part Champlain ne parle de ce voyage aux Gens de Mer; il est muet là-dessus dans les lettres qu'il écrit à Richelieu, dont il est à Québec le lieutenant: soucieux de renseigner le cardinal-ministre sur ce qui se fait d'important ici ${ }^{18}$, Champlain, que nul voyage d'exploration ne peut laisser indifférent, ne fait pas la moindre allusion au voyage de Nicollet. Une conclusion s'impose: Champlain n'est pas le patron de l'entreprise.

Il faut donc remonter encore en arrière. Rien n'empêche que le voyage ait été fait en 1632-1633, sous l'autorité du commandant Emery de Caën, mais rien ne le prouve non plus. Or les Relations des Jésuites utilisent une bien vague formule à propos de Nicollet: «il fut delegué»; pourquoi cette discrétion sur le haut responsable de l'ambassade? le voyage serait-il une initiative des Kirke? Un

16 Relation de $1634, \mathrm{RJ}, 7: 224-226$.

17 A. Gosselin, Jean Nicolet et le Canada de son temps (éd. 1905), 213-217.

18 Voir ses lettres à Richelieu en 1633 et 1634, dans Champlain, Oeuvres (éd. Biggar), app. vi et vii, dans le vol. VI: 375-379. 
mémoire anglais précise, en effet, que durant l'occupation de Québec les Anglais se sont rendus jusqu'à 400 lieues à l'intérieur des terres «for further discoveries» ${ }^{19}$ : ce qui, reporté sur la carte de Champlain en 1632, nous mène bien au-delà du lac des Hurons. Les Anglais ne pouvaient évidemment utiliser les routes de la fourrure qu'avec le concours des interprètes; et justement, Champlain affirme qu'un interprète des Algonquins, "Gros Jean de Dieppe», s'est mis au service des Kirke: ce "Gros Jean de Dieppe» serait-il Jean Nicollet ${ }^{20}$ ? Enfin, cette ambassade peut être le fait ni d'Emery de Caën ni des Kirke, mais tout simplement du grand Conseil de la Huronie: on voit Nicollet partir de la Huronie même et y revenir après sa mission ${ }^{21}$, tout comme s'il tenait ses titres de la Huronie. Quoi qu'il en soit, à moins qu'on ne découvre de nouveaux documents, on ne saura jamais en quelle année ni au service de qui Nicollet a accompli son long voyage.

\section{Une seule direction possible: le lac Supérieur}

Ei pour ce voyage, queì itinéraire Nicoilet a-t-il suivi? Pour le savoir, il nous faut suivre scrupuleusement ce que nous transmettent les jésuites Paul Le Jeune et Barthélemy Vimont, les seuls à nous renseigner sur le trajet de Nicollet. Pour sa part, le P. Le Jeune écrit: "Je diray en passant que le sieur Nicolet interprète en langue Algonquine \& Huronne, pour Messieurs de la nouvelle France ${ }^{22}$, m'a donné les noms de ces nations qu'il a visité luy mesme pour la pluspart dans leur pays.» ${ }^{23}$ Suivons donc le témoignage jésuite.

À partir de la Huronie (donc à l'angle sud-est de la baie Georgienne), ce témoignage nous fait monter à la rivière des Français, cette rivière qui sert de déversoir au lac des Népissingues. Continuant à longer la rive nord «de ceste mer douce» (c'est-à-dire le lac

19 Texte dans Champlain, Oeuvres (éd. Laverdière), III, Pièces justif., xxii: 22s.

20 Dans un discours au général Kirke, Champlain déclare que «Gros Jean de Dieppe» (il n'en donne pas le nom de famille) s'est mis au service des Anglais (Champlain, Oeuvres, éd. Biggar, VI: 108). On connaît à cette époque 4 interprètes à prénom Jean: Godefroy de Lintot, Manet, Nicollet et Richer. Le premier est de Lintot, pays de Caux, où se trouve Dieppe; Nicollet vend une terre de Hainneville, près de Cherbourg, en 1619, mais rien n'empêche que par la suite il soit devenu habitant de Dieppe. Nous ignorons d'où venaient Manet (mentionné par Sagard, Histoire du Canada, II: 517, comme étant interprète en 1626-1627) et Richer qui était chez les Népissingues en 1621-1624 (Sagard, op. cit., III: 336, 350s.) et chez les Algonquins peu après (Champlain, op. cit., VI: 108). Nous ignorons de même si Manet et Richer étaient encore au pays en 1629: on ne les y retrouve plus, en tout cas, à partir de 1632 .

21 Relation de 1642-1643, RJ, 23: 276-278.

22 Les Cent-Associés.

23 Relation de 1640, RJ, 18: 232. 
des Hurons), le P. Le Jeune mentionne les Amikoüai qui ont au sud une île d'une trentaine de lieues, où vivent les Outaouais: c'est notre île Manitouline, de quelque 80 milles de longueur. Nous sommes donc entre la rive nord du lac des Hurons et l'île Manitouline, dans ce qui porte aujourd'hui le nom de North Channel. De là, Le Jeune nous conduit au saut qu'habitent les Gens du Sault ou Saulteux, «pource qu'en effet il y a un Sault qui se jette en cet endroit dans la mer douce»: Le Jeune ne nomme pas ce saut, mais comme il n'y a pas de saut entre le lac Michigan et le lac des Hurons, mais seulement celui par où le lac Supérieur se déverse dans le lac des Hurons, nous voici, sans l'ombre d'un doute, au saut Sainte-Marie. Vers quelle direction va-t-on ensuite nous envoyer? «Au delà de ce sault, continue Le Jeune, on trouve le petit lac sur les bords duquel du costé du Nord sont les Roquai», qui eux-mêmes ont au nord les Mantoue. $A u$ delà de ce sault: nous voici donc en amont du saut Sainte-Marie, dans l'élargissement qui s'appelle aujourd'hui lac George, et nous avons toujours à notre droite les Amérindiens de la rive nord. Autre étape: «passant ce plus petit lac on entre dans la second mer douce». Nous ne pouvons vraiment pas être ailleurs que dans le lac Supérieur! Enfin, parlant toujours de cette seconde mer douce et de son littoral nord ${ }^{24}$, Le Jeune ajoute: «sur les rives de laquelle sont les Maroumine, plus avant encore sur les mesmes rives habitent les Ouinipigou peuple sedentaire qui sont en grand nombre» ${ }^{25}$.

Ainsi donc, selon Le Jeune qui écrit en 1640 en se fondant sur les conversations qu'il a eues avec Nicollet, les Ouinipigous que cet interprète est allé rencontrer, sont établis sur la rive nord du lac Supérieur.

\section{Les Ouinipigous, algonquiens du lac Supérieur}

En localisant en 1640 les Ouinipigous sur la rive nord du lac Supérieur, là où quelque dix ans plus tôt Nicollet s'était rendu, Le Jeune confirme, à un détail près, l'information que Champlain donnait sur ses cartes de 1616 et de 1632, à savoir que les Ouinipigous ou Puants sont au nord des Grands Lacs, information que l'on a pris l'habitude de considérer comme erronée, parce qu'on tenait à situer les Ouinipigous de Nicollet dans le lac Michigan. $\grave{A}$ un détail

24 À la fin du paragraphe qui précède celui que nous citons, Le Jeune annonce qu'il va énumérer des nations «qui bordent ces grands Lacs ou ces mers du costé du Nord» (Relation de 1640, RJ, 18: 228); plus loin, il annonce qu'il énumérera les nations du sud (ibid., 232): ce qu'il fait aux pages $232-234$.

25 Relation de 1640, RJ, 18: 228. 
près, disons-nous, car Champlain en 1616 et en 1632 situe les Ouinipigous au pied du saut Sainte-Marie au lieu de les représenter en amont du saut. Sur sa carte de $1616^{26}$, Champlain qui a passé l'hiver précédent en Huronie, mais sans visiter la rive nord des Grands Lacs, a dessiné à l'angle nord-ouest du lac des Hurons, une rivière qui vient du nord se déverser au pied du saut et, à gauche de cette rivière, il a écrit Les puans. Dans sa carte de $1632^{27}$, un peu mieux renseigné, il représente au même endroit un lac d'une forme quasi ronde, avec cette inscription La Nation des Puans; au milieu de ce lac, une île sur laquelle il donne l'explication suivante: Isle ou il y a une mine de cuivre; ce lac se déverse vers le sud par une rivière dont Champlain écrit dans la Table qui accompagne la carte: Riviere des Puans, qui vient d'un lac auquel il y a une mine de Cuivre de rosette ${ }^{28}$. Ce lac de la Nation des puans ou Ouinipigous rappelle étrangement par sa forme et par ses proportions le lac appelé autrefois lac des Alemenipigons ${ }^{29}$ et dit aujourd'hui lac Nipigon, mais Champlain le fait se déverser dans l'angle nord-ouest du lac des Hurons au ỉeu de ie faire se déverser dans le lac Supérieur.

Mais qui sont donc ces Ouinipigous ou Puants que les Relations fixent comme objectif à la mission de Nicollet dans le lac Supérieur? Le Jeune écrit en 1640: "Quelques François les appellent la Nation des Puans, à cause que le mot Algonquin ouinipeg signifie eau puante. Or ils nomment ainsi l'eau de la mer salée, si bien que ces peuples se nomment Ouinipigou, pource qu'ils viennent des bords d'une mer dont nous n'avons point de cognoissance, \& par consequent il ne faut pas les appeller la nation des Puans, mais la

26 Découverte en 1953 et conservée aux Archives publiques du Canada, cette carte couvre la même superficie que celle de 1632, mais elle n'est pas terminée; elle est d'une précision particulière dans sa représentation de la rivière des Outaouais, du lac des Népissingues, de la rivière des Français, ainsi que du lac Ontario: sur cette carte. voir un article de Conrad Heidenreich, "An Analysis of Champlain's Maps», 12-45, dans Écrits de voyage re latifs à la Nouvelle-France, publiés par Jacques Cotnam et Jack Warwick, aux Editions de l'Université d'Ottawa, 1978.

27 Carte reproduite dans notre Atlas de la Nouvelle-France, $86 \mathrm{~s}$. Sur cette carte, voir aussi notre étude La carte de Champlain en 1632: ses sources et son originalité (Québec, les Presses de l'Université Laval, 1978, cahier No 51 de la collection Cartologica); aussi de Heidenreich, Explorations and Mapping of Samuel de Champlain, 1603-1632 (Cartographica, Monograph No. 17, 1976); et, du même auteur, l'étude citée à la note précédente.

28 Pour la Table de cette carte de 1632, voir Champlain, Oeuvres (éd. Biggar), VI: 224-252. Sur ce commentaire de Champlain, l'éditeur Biggar ne manque pas de faire remarquer qu'il y a ici erreur, les Puants selon lui devant se situer plutôt dans le lac Michigan: pourtant, Le Jeune est bien explicite là-dessus en 1640.

29 Franquelin en 1684 donne Lac Alemenigon (voir notre Atlas de la Nouvelle-France, 94); Bellin en 1755 a Lac Alimipegon (ibid., 120); une carte de 1783 (ASQ, tiroir 220; 3) se lit Lac Alemipigon. 
nation de la mer ${ }^{30}$ : d'où le nom de Gens de Mer que l'on rencontre sous la plume de Le Jeune et ailleurs pour désigner les Ouinipigous, et l'on trouve même l'expression peuples maritimes ${ }^{31}$. La Relation de 1647-1648 précise qu'ils étaient venus de la Mer du Nord: «ils se disent estre venus des costes d'une mer fort éloignée, vers le Septentrion» et dont l'eau est salée ${ }^{32}$.

Autre problème de haute importance: la langue que parlent ces Ouinipigous. Des nations que Nicollet a visitées, Le Jeune écrit: «tous ces peuples entendent l'Algonquin, excepté les Hurons, qui ont une langue à part, comme aussi les Ouinipigou ou gens de mer ${ }^{33}$; phrase qui n'est pas très claire, mais que la Relation de 1647-1648 vient expliciter: les Ouinipigous sont d'une langue «inconnu», c'est à dire qui n'est ny Algonquine, ny Hurone». ${ }^{34}$. On a donc été très heureux, comme l'a fait l'historien Shea, suivi par tous les autres, de pouvoir en toute confiance identifier les Ouinipigous à la nation des Winnebagoes qui vivaient sur les bords du lac Michigan et qui, étant de famille siouse, ne parlaient ni algonquin ni huron. Or un détail du récit de Nicollet vient démolir cette thèse et, en même temps, nous faire mieux comprendre ce que voulaient dire les écrivains jésuites: lorsque Nicollet eut prévenu les Gens de Mer de son arrivée, ceux-ci dépêchèrent des jeunes gens pour aller au-devant de celui qu'ils surnommaient Manitouiriniou, c'est-à-dire homme merveilleux ${ }^{35}$. Comme le démontre facilement l'historien Dever ${ }^{36}$, en se fondant sur les connaissances acquises depuis le siècle dernier, Manitouiriniou, c'est de l'algonquien et non du sioux; et si les Ouinipigous décorent Nicollet de ce titre, c'est qu'ils sont de famille algonquienne. Ce qui ne vient nullement en contradiction avec les Relations: au XVIIe siècle, on n'a pas encore le concept algonquien ou algique pour grouper en une même famille ces nations amérindiennes qui ont entre elles une communauté culturelle et dont les langues, quoique distinctes, ont une même origine. Quand les jésuites Le Jeune et Vimont écrivent que

30 Relation de 1640, RJ, 18: 230. La Relation de 1647-1648 (RJ, 33: 150) donne la même origine. Toutefois, à propos de la baie des Puants (celle du lac Michigan), le jésuite Thierry Beschefer nuance l'explication en 1683: baie «que Lon appelle des puans pour n'avoir pas bien entendu [compris]. Le mot sauvage qui signifie des eaux qui sentent Le Jonc» (lettre au Provincial, 21 oct. 1683, RJ, 62: 202).

31 Relation de 1659-1660, RJ, 46: 68 .

32 Relation de 1647-1648, RJ, 33: 150.

33 Relation de 1640, RJ, 18: 232.

34 Relation de 1647-1648, RJ, 33: 150.

35 Relation de 1642-1643, RJ, 23: 276. 318-322.

36 Harry Dever, The Nicolet Myth, dans Michigan History, 50, 4 (December 1966): 
les Ouinipigous de Nicollet ne parlent ni l'algonquin ni le huron, ils entendent par algonquin la langue des Algonquins de l'île aux Allumettes et du Saint-Laurent; mais d'autres peuples, parce qu'ils étaient de famille algonquienne, parlaient une langue apparentée à l'algonquin: entre autres, les Népissingues, les Outaouais, les Ojibwés (dont les Saulteux) et, dans le lac Supérieur, les Kilistinons ou Cristinaux qui sont nos Cris d'aujourd'hui. Ce Manitouiriniou, dont les Ouinipigous gratifient Nicollet, atteste bien que, même s'ils ne parlent pas comme les Algonquins, ils sont de famille algonquienne et non de famille siouse.

\section{Un bouleversement ethnique qui explique la confusion}

Comment, avec des éléments tirés de la seule source existante d'information, les historiens ont-ils pu envoyer Nicollet dans le lac Michigan et jusque vers la rivière Wisconsin, quand on sait, en outre, toujours par les mêmes Relations des Jésuites que le lac Michigan n'est encore connu en 1648 que par ouï-dire ${ }^{37}$ et quand on sait avec précisioñ quie les prentiers Français n'y entrent qu'en $1669^{38}$ !

37 Ce qui est manifeste dans la Relation huronne du jésuite Ragueneau (RJ, 33: 148-150). De même, les deux cartes de Sanson d'Abbeville en 1656 et du jésuite Du Creux en 1660 ne représentent le lac Michigan que sur des renseignements fournis par des Amérindiens: voir la carte de Sanson d'Abbeville dans notre Atlas de la Nouvelle-France (88) et celle de Du Creux dans RJ, 46 (front.)

38 Lorsque le jésuite Alloüez entre dans le lac Michigan, le 4 novembre 1669, il écrit que ce lac est «inconnu jusques à present» (Relation de 1669-1670, RJ, 54: 198). Or Alloüez missionnait dans les Grands Lacs depuis 1665. Par ailleurs, ce que démontrent les Relations, les Jésuites étaient constamment au courant de tout ce qui se faisait d'exploration dans les Grands Lacs; on sait, en particulier, qu'ils sont restés en relations étroites avec leur ancien serviteur de la Huronie, Chouart des Groseillers, cet explorateur des années 1654-1656 et 1659-1660.

L'historien Heidenreich est intervenu récemment avec une thèse pour le moins surprenante. Suivant la thèse traditionnelle qui veut que les Puants, du temps de Champlain, vivent dans le lac Michigan, Heidenreich soutient que la grande mer dessinée par Champlain en 1632 à l'ouest du lac des Hurons est non pas le lac Supérieur, mais le lac Michigan: ses conclusions résultent d'une étude des longitudes et des latitudes de la carte de 1632 (voir cette étude dans Explorations and Mapping, plus haut cité, note 27). S'il y avait chez Champlain une utilisation consistante des mesures, on pourrait tenter de confirmer cette thèse par les autres sources écrites de la même époque; or cette utilisation des mesures est tout à fait inconsistante chez Champlain, comme le démontre Heidenreich lui-même (voir son article "An Analysis of Champlain's Maps», plus haut cité note 26). Par ailleurs, les sources écrites de cette époque identifient trop clairement le lac Supérieur pour qu'on hésite à le reconnaître: 1 le Sault de Gaston qui, selon Champlain (Oeuvres, éd. Biggar, VI: 234), «se descharge dans la mer douce, venant d'un autre grandissime lac», ne peut que se rapporter au saut SainteMarie à la sortie du lac Supérieur, car on sait très bien qu'il n’y a pas de saut entre le lac Michigan et le lac des Hurons; 2- la Relation de 1640, qui décrit la direction prise par Nicollet, et ce d'après la déclaration même de Nicollet, ne mène nulle part qu'à la rive nord du lac Supérieur; 3- les Ouinipigous ou Puants que rencontre Nicollet sont de langue algonquienne et non de langue siouse; 4- la Relation de 1669-1670 affirme que les premiers Français n'entrent dans le lac Michigan qu'en 1669. 
L'explication de cette confusion se trouve dans le grand bouleversement qui change le paysage ethnique des Grands Lacs dans le deuxième quart du XVIIe siècle. Voulant localiser les Ouinipigous de Nicollet dans les années 1630, les historiens qui ont battu la fausse piste, se sont principalement fondés sur les cartes de Sanson d'Abbeville en 1656 et du jésuite Du Creux en 1660, qui situent les Ouinipigous ou Puants dans le lac Michigan ${ }^{39}$ : ces historiens en ont conclu que si les Ouinipigous sont là en 1660 et en 1656, ils étaient dans ce même lac du temps de Nicollet et de Champlain.

Ils ont ainsi négligé un fait important qui survient dans l'intervalle qui sépare Nicollet et les cartographes Sanson d'Abbeville et Du Creux: le grand bouleversement ethnique produit dans les Grands Lacs par les guerres amérindiennes, dans le deuxième quart du siècle. Les Algonquins de l'île aux Allumettes, par exemple, ont été forcés de se transporter ailleurs, dont certains sur la rive nord du lac Supérieur et dans le lac Michigan: ils y sont assez nombreux au milieu du siècle pour que Du Creux appelle le lac Michigan grand lac des Algonquins; les Hurons ont été détruits ou sont allés se réfugier soit du côté de Québec soit du côté du Mississipi; les Outaouais sont passés en bon nombre dans le lac Supérieur; les Pétuns se sont retirés dans le lac Michigan et au-delà: on en rencontrera à l'extrémité occidentale du lac Supérieur.

Dans des circonstances mal connues, les Ouinipigous ou Puants ont subi le même bouleversement. Habitant, à l'origine, la mer du Nord ${ }^{40}$, ils s'étaient établis sur la rive nord du lac Supérieur: c'est là que les Jésuites, les seuls à nous renseigner sur le voyage de Nicollet, les situent en $1640^{41}$. De là, peut-être pour s'éloigner d'autres nations algonquiennes avec qui ils sont en guerre, car la paix ménagée par Nicollet fut de courte durée $e^{42}$, ils vont résider pendant un certain temps dans la péninsule dite aujourd'hui Michigan qui est entre le lac des Hurons et le lac Michigan ${ }^{43}$ : en 1647-1648, ils sont

39 Dans la carte de Sanson d'Abbeville, le lac Michigan (seulement esquissé) est appelé Lac des Puans. Du Creux ne fait lui aussi qu'esquisser ce lac et il inscrit: Magnus Lacus Algonquinorum seu Lacus Foetentium, c'est-à-dire Grand lac des Algonquins ou lac des Puants.

40 Relation de 1647-1648, RJ, 33: 150; de 1669-1670, RJ, 54: 134.

41 Relation de 1640, RJ, 18: 228-230.

42 Relation de 1636, RJ, 10: 82 . On y affirme que la guerre a repris en 1635 entre Algonquins et Gens de Mer.

43 L'auteur de la Relation de 1670-1671 écrit, de l'île de Michillimackinac, que les Puants avaient déjà résidé «à la terre ferme qui est au midy de cette Isle»; plus loin, il fait allusion aux terres qu'ils occupaient «entre le Lac des Hurons, \& celui des Illinois», c'est-àdire le lac Michigan; puis, ils en furent chassés et se réfugièrent «comme étrangers» dans la baie des Puants $(158,182)$. 
à la fois sur le littoral est de cette péninsule et à l'intérieur du lac Michigan, qui reçoit alors le nom de lac des Puants, où sont aussi des Pétuns et des Outaouais ${ }^{44}$; ce qui explique que Sanson d'Abbeville en 1656 inscrive Lac des Puans sur le lac Michigan; et ce qui explique que la Relation de 1659-1660 désigne ce lac Michigan par le nom de lac des peuples maritimes, les Puants étant dits Gens de Mer, et que Du Creux en 1660 écrive du lac Michigan: Grand lac des Algonquins ou lac des Puants ${ }^{45}$. Finalement, ils vont évacuer la péninsule du Michigan pour se retrouver dans une large baie de la rive occidentale du lac Michigan, baie qui sera dite baie des Puants, puis lac Saint-François et Baie Verte, aujourd'hui Green Bay ${ }^{46}$. Ce que deviennent ensuite ces Ouinipigous ou Puants de famille algonquienne qui résident «comme étrangers» dans le lac Michigan ${ }^{47}$ et comment l'historien Shea a pu leur substituer les Winnebagoes, ne sont plus de notre propos.

\section{Saura-t-on jamais jusqu'où Nicollet s'est rendu?}

Selon les indications qu'il a lui-même données au jesuite Le Jeune, Nicollet aurait donc remonté au-delà du saut Sainte-Marie pour entrer dans le lac Supérieur et, en longeant la rive nord, il aurait rencontré les Ouinipigous: nous ignorons sur quel point précis de la rive nord, parce que les Relations ne nous donnent aucun point de repère, mais il est tout naturel de penser qu'il les a rejoints dans ce lac des Puants où ils vivaient, selon Champlain, et qui nous paraît bien être l'actuel lac Nipigon. De même, il est impossible de connaître les explorations que Nicollet a pu faire au cours de son ambassade. Parce que, croyait-on, ces Gens de Mer vivaient près de la mer d'Asie (du temps de Nicollet, on la supposait non loin au-delà du lac des Hurons), l'interprète avait mis dans ses bagages «une grande robbe de damas de la Chine, toute parsemée de fleurs, $\&$ d'oyseaux de diverses couleurs»: c'est revêtu de ce costume de mandarin qu'il prit part à une grande assemblée de quelques milliers d'hommes, où l'on fit festin pour conclure la paix entre Ouinipigous et Algonquins ${ }^{48}$.

44 Relation de 1646-1648, RJ, 33: 148-150.

45 Relation de 1659-1660, RJ, 46: 68; pour les cartes, voir plus haut note 40.

46 Voir la Relation de 1669-1670, RJ, 54: 216. Pour désigner le lac Michigan, cette "troisième mer douce», on dit d'abord lac des Puants ou des Algonquins; quand les Français y pénètrent en 1669, ils disent lac des Illinois, puis lac Saint-Joseph (récit du jésuite Alloüez, 1677, RJ, 60: 152).

47 Relation de 1670-1671, RJ, 55: 158.

48 Relation de 1642-1643, RJ, 23: 276-278. On dit ici que l'assemblée fut de 4000 ou 5000 personnes; dans la Relation de 1655-1656 (RJ, 42: 222), on parle de 3000. 
Costume qui montre bien la préoccupation de Nicollet de trouver la mer d'Asie. Or, rapporte Le Jeune, si Nicollet «eust vogué trois jours plus avant sur un grand fleuve qui sort de ce lac», il «auroit trouvé la mer» et Le Jeune de conclure: «j'ay de fortes conjectures que c'est la mer qui respond au Nord de la nouvelle Mexique, \& que de cette mer, on auroit entrée vers le Japon \& vers la Chine ${ }^{49}$. On ne connaît aucun grand fleuve qui sort ainsi du lac Supérieur: Nicollet aura mal compris les Ouinipigous qui lui parlaient d'une rivière par où on sort de ce lac. De ces rivières il n'en manque pas dans la région du lac Supérieur: du lac Nipigon on atteint rapidement les grandes voies d'eau qui vont se déverser dans la baie d'Hudson; à l'extrémité occidentale du lac Supérieur, une route d'eau mène à l'immense plan d'eau du lac Winnipeg; et non loin de l'actuelle Duluth, on atteint cette rivière Sainte-Croix qui conduit au Mississipi, dont le nom algonquien signifie grande rivière $^{50}$. Les tenants de la thèse du lac Michigan ont entassé hypothèses sur hypothèses pour tenter de suivre avec précision la route de Nicollet et de calculer les distances qu'il aurait parcourues: puisque c'est dans le lac Supérieur qu'il est allé, faut-il recommencer toutes les spéculations, à partir des mêmes éléments très vagues? quelle est cette voie de communication par laquelle il aurait pu en trois jours trouver une mer, et quand il a compris mer, de quelle grande étendue d'eau s'agissait-il: la baie d'Hudson que les cartes d'alors montraient s'ouvrant vers l'Asie? ou bien le bassin du lac Winnipeg? Aussi longtemps qu'on ne découvrira de nouveaux documents, les plus habiles dissertations ne produiront pas une parcelle de vérité.

Que Nicollet soit allé non pas dans le lac Michigan, mais dans le lac Supérieur et que, même dans cette dernière région, il ne soit pas le premier à pénétrer, Étienne Brûlé l'y ayant probablement devancés1, n'enlève pas pour autant le prestige de son aventure. Certes, on ne sait pas jusqu'où il s'est rendu dans ce lac Supérieur et on ne le savait pas davantage quand on lui attribuait la «décou-

49 Relation de 1640, RJ, 18: 236.

50 Jean Delanglez, Louis Jolliet, 77.

51 Sagard écrit: "Le Truchement Bruslé avec quelques Sauvages nous ont asseuré qu'au delà de la mer douce, il y a un autre grandissime lac, qui se descharge dans icelle par une cheute d'eau que l'on a surnommé le Saut de Gaston» (Histoire du Canada, éd. Tross, III: 589): même si Sagard, qui publie en 1636, a pu retenir de la Table de Champlain le détail concernant le "grandissime lac» et le nom du saut, rien n'empêche de croire qu'en 1623-1624 il ait pu causer de tout cela avec Brûlé; et puisque Brûlé a pu tenter une évaluation de la longueur du lac, on peut penser qu'il a pénétré dans ce lac. 
verte» du lac Michigan et du Wisconsin. Un fait toutefois demeure certain, si l'on s'en tient aux Jésuites du XVIIe siècle qui s'y connaissaient en explorations: le jésuite Paul Le Jeune en 1640 salue en Nicollet l'homme «qui a le plus avant penetré dedans ces pays si esloignés $\aleph^{52}$. Et cela suffit pour qu'après les Ouinipigous on le qualifie de Manitouiriniou... 